\title{
INTERAÇÃO ENTRE NEMATICIDAS E HERBICIDAS PRÉ- EMERGENTES NO DESENVOLVIMENTO INICIAL DA CULTURA DA CANA-DE-AÇÚCAR
}

\author{
Fábio Akira Taira ${ }^{1}$ \\ José Vinicius Carraschi \\ Anderson Cleiton Quinaia ${ }^{3}$ \\ Adão Izidoro Junior ${ }^{4}$ \\ Elizeu Junior da Silva ${ }^{5}$ \\ Raiane Pereira Schwengber ${ }^{6}$ \\ Ana Daniela Lopes ${ }^{7}$ \\ Juliana Silveira do Valle ${ }^{8}$ \\ Simone de Melo Santana Gomes ${ }^{9}$
}

TAIRA, F. A.; CARRASCHI, J. V.; QUINAIA, A. C.; IZIDORO JUNIOR, A.; SILVA, E. J. da; SCHWENGBER, R. P.; LOPES, A. D.; VALLE, J. S. do; GOMES, S. de M. S. Interação entre nematicidas e herbicidas pré-emergentes no desenvolvimento inicial da cultura da cana-de-açúcar. Arq. Ciênc. Vet. Zool. UNIPAR, Umuarama, v. 20, n. 2, p. 57-63, abr.jun. 2017.

RESUMO: O objetivo do trabalho foi avaliar a interação do herbicida tebuthiuron (Combine ${ }^{\circledR} 500 \mathrm{SC}$ ), com nematicidas carbofuram (Furadan ${ }^{\circledR} 350$ SC) e benfuracarbe (Pottente $\left({ }^{\circledR}\right)$, aplicados no plantio da cultura da cana-de-açúcar (RB 867515). O experimento foi conduzido em casa de vegetação da Universidade Paranaense, em Umuarama - PR, no período de abril a agosto de 2014. Foi instalado em vasos com capacidade para 4,5 L, contendo uma planta por vaso, em delineamento inteiramente casualizado, com seis tratamentos e quatro repetições, sendo os tratamentos testemunha $\left(\mathrm{T}_{1}\right)$, sem aplicação de produtos; aplicação isolada de carbofuran $\left(\mathrm{T}_{2}\right)$; aplicação isolada de benfuracarbe $\left(\mathrm{T}_{3}\right)$; aplicação isolada de tebuthiuron $\left(\mathrm{T}_{4}\right)$; aplicação combinada de carbofuran e tebuthiuron $\left(\mathrm{T}_{5}\right)$ e aplicação combinada de benfuracarbe e tebuthiuron $\left(\mathrm{T}_{6}\right)$. $\mathrm{O}$ experimento foi submetido a duas avaliações, 45 e 90 dias após o plantio (DAP) sendo que na primeira, as variáveis avaliadas foram altura da parte aérea, número de folhas e número de plantas daninhas por vaso, e, na segunda, altura da parte aérea; número de folhas; número de plantas daninhas; massa fresca e seca da parte aérea; massa fresca do sistema radicular; população final total de nematoides por vaso, calculada pelo somatório de nematoides nas raízes e solo. Quanto ao número de folhas, o tratamento $\mathrm{T}_{4}$ demonstrou fitotoxicidade aos 45 DAP. Os tratamentos $\mathrm{T}_{3}, \mathrm{~T}_{5}$ e $\mathrm{T}_{6}$ apresentaram as maiores massa fresca e seca da parte área da cana, demonstraram ausência de interação antagônica entre as combinações do herbicida com os nematicidas aos 90 DAP. Os tratamentos $\mathrm{T}_{3}$ e $\mathrm{T}_{5}$ foram eficientes no controle populacional de P. zeae.

PALAVRAS-CHAVE: Benfuracarbe. Carbofuran. Combinação. Tebuthiuron. Saccharum spp.

\section{INTERACTION BETWEEN NEMATICIDES AND PRE-EMERGENT HERBICIDE IN THE INITIAL DEVELOPMENT OF SUGAR CANE}

\begin{abstract}
The aim of the study was to evaluate the interaction of the herbicide tebuthiuron (Combine ${ }^{\circledR}$ SC 500), with the following nematicides: carbofuran ( 350 Furadan ${ }^{\circledR} S C$ ) and benfuracarb (Pottente ${ }^{\circledR}$ ) applied during the planting of cane sugar (RB 867515). The experiment was conducted in a greenhouse at Universidade Paranaense in Umuarama - PR, from April to August 2014. The crop was started in 4.5-L vases containing one plant per vase, in a completely randomized design with six treatments and four replications, namely control treatments $\left(\mathrm{T}_{1}\right)$, with no application of products; isolated application of carbofuran $\left(\mathrm{T}_{2}\right)$; isolated application of benfuracarb $\left(\mathrm{T}_{3}\right)$; isolated application of tebuthiuron $\left(\mathrm{T}_{4}\right)$; combined application of carbofuran and tebuthiuron $\left(\mathrm{T}_{5}\right)$ and combined application of benfuracarb and tebuthiuron $\left(\mathrm{T}_{6}\right)$. The experiment was submitted to two assessments, at 45 and 90 days after planting (DAP). On the first assessment, the variables analyzed were shoot height, number of leaves and number of weeds per vase. The second assessment analyzed shoot height; number of leaves; number of weeds; fresh and dry weight of shoot; fresh weight of the root system; total final population of nematodes per vase, calculated by the sum of nematodes in the roots and soil. Regarding the number of leaves, the $\mathrm{T}_{4}$ treatment showed phytotoxicity at 45

DOI: 10.25110 /arqvet.v20i2.2017.6150

${ }^{1}$ Engenheiro agrônomo, Umuarama, PR, Brasil. E-mail: taira@usacucar.com.br.

${ }^{2}$ Engenheiro agrônomo, Umuarama, PR, Brasil. E-mail:viniciuscarraschi@hotmail.com

${ }^{3}$ Engenheiro agrônomo, Umuarama, PR, Brasil. E-mail:ac.quinaia@hotmail.com

${ }^{4}$ Graduando em Engenharia Agronômica, Universidade Paranaense, Umuarama, PR, Brasil. E-mail: adao_1995@hotmail.com

${ }^{5}$ Programa de Pós-Graduação em Ciências Agrárias, Universidade Estadual de Maringá, Umuarama, PR, Brasil. E-mail: elizeu-junior17@hotmail.com

${ }^{6}$ Graduando em Engenharia Agronômica, Universidade Paranaense, Umuarama, PR, Brasil. E-mail: raiane schwengber@hotmail.com

${ }^{7}$ Docente do Programa de Pós-graduação em Biotecnologia Aplicada à Agricultura, Universidade Paranaense, Umuarama, PR, Brasil. E-mail: anadanielalopes@prof.unipar.br

${ }^{8}$ Docente do Programa de Pós-graduação em Biotecnologia Aplicada à Agricultura, Universidade Paranaense, Umuarama, PR, Brasil. E-mail: jsvalle@prof. unipar.br

${ }^{9}$ Programa de Pós-graduação em Biotecnologia Aplicada à Agricultura, Universidade Paranaense, Umuarama, PR, Brasil. E-mail: simonemelo@prof. unipar.br
\end{abstract}


DAP. The $\mathrm{T}_{3}, \mathrm{~T}_{5}$ and $\mathrm{T}_{6}$ treatments had the highest fresh and dry weight of the aerial part of the sugar cane; with absence of antagonistic interaction between the combinations of herbicide and nematicides at $90 \mathrm{DAP}$. The $\mathrm{T}_{3}$ and $\mathrm{T}_{5}$ treatments were effective in controlling the population of P. zeae.

KEYWORDS: Benfuracarbe. Carbofuran. Combination. Tebuthiuron. Saccharum spp.

\section{INTERACCIÓN ENTRE NEMATICIDAS Y HERBICIDAS PRE EMERGENTES EN EL DESARROLLO INICIAL DE LA CULTURA DE CAÑA DE AZÚCAR}

RESUMEN: El objetivo del estudio ha sido evaluar la interacción del herbicida tebuthiuron (Combine ${ }^{\circ} 500$ SC), con nematicidas carbofuram (Furadan ${ }^{\circledR} 350$ SC) y benfuracarbe (Pottente $\left.{ }^{\circledR}\right)$, aplicados en plantío de caña de azúcar (RB 867515). El experimento ha sido dirigido en el área de vegetación de la Universidad Paranaense, en Umuarama - PR, en el período de abril a agosto de 2014. Se ha instalado en floreros con capacidad para 4,5 L, conteniendo una planta por florero, en delineamiento enteramente casualizado, con seis tratamientos y cuatro repeticiones, siendo los tratamientos testigo $\left(\mathrm{T}_{1}\right)$, sin aplicación de productos; aplicación aislada de carbofuran $\left(\mathrm{T}_{2}\right)$; aplicación aislada de benfuracarbe $\left(\mathrm{T}_{3}\right)$; aplicación aislada de tebuthiuron $\left(\mathrm{T}_{4}\right)$; aplicación combinada de carbofuran y tebuthiuron $\left(\mathrm{T}_{5}\right)$ y aplicación combinada de benfuracarbe y tebuthiuron $\left(\mathrm{T}_{6}\right)$. El experimento ha sido sometido a dos evaluaciones, 45 y 90 días después del plantío (DAP) siendo que en la primera, las variables evaluadas fue la altura de la parte aérea, número de hojas y el número de plantas dañinas por florero, y, en la segunda, altura de la parte aérea, número de hojas, número de plantas dañinas; masa fresca y seca de la parte aérea; masa fresca del sistema radicular; población final total de nematodos por florero, calculado por la suma de nematodos en las raíces y suelo. Cuanto al número de hojas, el tratamiento $\mathrm{T}_{4}$ demostró citotoxicidad a los 45 DAP. Los tratamientos $\mathrm{T}_{3}, \mathrm{~T}_{5} \mathrm{y}_{6}$ presentaron mayor masa fresca y seca en la parte aérea de la caña, demostraron ausencia de interacción antagónica entre las combinaciones de los herbicidas con los nematicidas a los 90 DAP. Los tratamientos $\mathrm{T}_{3} \mathrm{y}_{5}$ fueron eficientes en el control poblacional de P. zeae.

PALAVRAS CLAVE: Benfuracarbe. Carbofuran. Combinación. Tebuthiuron. Saccharum spp.

\section{Introdução}

A cana-de-açúcar (Saccharum officinarum) foi a primeira cultura de interesse econômico cultivado no Brasil. A área destinada à atividade sucroalcooleira na safra 2013/2014 foi estimada em 8.810,79 milhões de hectares, sendo o estado de São Paulo o maior produtor, responsável por $51,66 \%$ da produção nacional, ultrapassando os estados de Goiás, Minas Gerais, Paraná, Mato Grosso do Sul, Alagoas e Pernambuco, com produtividade média, nestas regiões, de 74,8 $\mathrm{tha}^{-1}$ (CONAB, 2013).

Dentre os fatores que afetam acentuadamente a produtividade da cana-de-açúcar, a incidência de plantas daninhas e a ocorrência de nematoides merecem destaque. Em áreas infestadas com nematoides, as perdas podem chegar a mais de 50\%, no caso das espécies Meloidogyne incognita e Pratylenchus zeae (NOVARETTI, 1981.; DINARDO-MIRANDA.; FERRAZ, 1991). Da mesma forma, as plantas daninhas interferem negativamente, pois podem competir por recursos limitantes do meio (principalmente água, luz e nutrientes), liberar substâncias alelopáticas e assim inibir a brotação da cana-de-açúcar, hospedar pragas e doenças comuns à cultura ou, ainda, interferir no rendimento da colheita (PITELLI; DURIGAN, 1985). Segundo Procópio et al. (2003), a interferência das plantas daninhas pode promover perdas na produtividade da lavoura em níveis que variam de 10 a $80 \%$.

Quanto ao manejo desses fatores limitantes, o controle químico de nematoides com nematicidas tem-se mostrado uma excelente opção. Em geral, esses produtos podem eliminar até $90 \%$ da população de nematoides de uma área, e quando empregados corretamente, tem proporcionado resultados altamente compensatórios. Em estudos nos quais se aplicaram nematicidas no plantio de diversas variedades, cultivadas em campos infestados por uma ou mais espécies desses parasitos, foram observados incrementos de produtividade agrícola no primeiro corte, em relação às testemunhas, de até $41 \mathrm{t} \mathrm{ha}^{-1}$ (DINARDO-MIRANDA et al., 1995; DINARDO-MIRANDA et al., 1996; DINARDO-MIRANDA et al., 1998; GARCIA et al., 1997).

$\mathrm{Na}$ cultura da cana-de-açúcar, o controle químico com nematicidas e herbicidas tem contribuído significativamente para manutenção da produtividade agrícola. A aplicação sistemática de nematicidas no sulco de plantio e, logo após, a pulverização de herbicidas em condição de pré-emergência, ocorrem de forma rotineira nas áreas produtoras. Quando as práticas agrícolas recomendadas são seguidas, esses compostos químicos não são prejudiciais individualmente, porém a tolerância das plantas a um determinado defensivo pode ser alterada pela presença de outro. Uma cultura tolerante a um herbicida pode ter essa capacidade reduzida devido à interação sinérgica entre o herbicida e outro produto aplicado, como um nematicida, por exemplo (DINARDO-MIRANDA et al., 2001a).

Trabalhos de Blanco et al. (1980a) e Copersucar (1982a) já demonstraram essa fitotoxicidade. Porém, os resultados ainda são contraditórios, como apontado por Romão (2008a), sugerindo que há muitos fatores, além da combinação nematicida e herbicida, que interferem na interação dos produtos em cana-de-açúcar, dentre os quais se destacam as variedades, tipo de solo e volume de chuvas após aplicação. Com base nesses resultados, há a necessidade de mais estudos a respeito das interações.

Dessa forma, o presente trabalho tem como objetivo avaliar a interação dos nematicidas carbofuran ${ }^{1}$ e benfuracarbe $^{2}$ aplicados no sulco de plantio, com o herbicida tebuthiuron $^{3}$ aplicado em pré-emergência, durante o período de de-

${ }^{1}$ Furadan ${ }^{\circledR} 350$ SC - Inseticida Nematicida Sistêmico do grupo químico Metilcarbamato de Benzofuranila, 100 Niagara Street - Middleport, NY EUA.

${ }^{2}$ Pottente ${ }^{\circledR}$ - Inseticida Nematicida, Sistêmico do grupo químico Metilcarbamato de Benzofuranila, 615 Hanamen, Satoura-cho, Tokushima 7728601, Japão.

${ }^{3}$ Combine ${ }^{\circledR} 500 \mathrm{SC}$ - Herbicida Seletivo, de ação sistêmica, do grupo quí- 
senvolvimento inicial da cultura da cana-de-açúcar.

\section{Materiais e Métodos}

O experimento foi conduzido em casa de vegetação da Universidade Paranaense - UNIPAR, Campus III, no município de Umuarama, Paraná. Para o experimento utilizou-se de gemas pré-germinadas de cana-de-açúcarvariedade RB 86 7515. Em Abril de 2014 foi realizado o tratamento pré-germinativo, para tanto, foram coletados colmos e divididos em toletes de aproximadamente $3 \mathrm{~cm}$, de forma a conterem apenas uma gema. Os toletes foram acondicionados em caixas plásticas transparentes do tipo "gerbox", contendo algodão umedecidocom água na proporção de 2,5 vezes a massa seca do algodão.

Posteriormente, as caixas plásticas foram mantidas umedecidas em estufa incubadora do tipo BOD (Demanda Bioquímica de Oxigênio) com luz constante e temperatura em torno dos $30^{\circ} \mathrm{C}$, durante 15 dias. Após este período, os toletes pré-germinados foram selecionados e transplantados para vasos plásticos com capacidade para 4,5 L, sendo que em cada unidade experimental foi plantado um tolete pré-germinado.

O solo utilizado é classificado como Latossolo Vermelho Distrófico Argissólico (EMBRAPA, 2013), de textura média, proveniente do município de Herculândia, Paraná, que estava naturalmente infestado por nematoides da espécie Pratylenchus zeae. A calagem e a adubação de implantação foram realizadas utilizando-se $1,95 \mathrm{~g}$ de calcário dolomítico e um grama de adubo formulado 02-16-06 de NPK, por unidade experimental, respectivamente, conforme análise química do solo.

O experimento foi constituído por seis tratamentos, sendo eles: $T_{1}$ - Testemunha, sem aplicação de inseticida/nematicida e/ou herbicida; $T_{2}$ - Aplicação de inseticida/nematicida a base de carbofuran, na dose de $2,345 \mathrm{Kg}$ de ingrediente ativo (i.a.) por hectare (ha); $\mathrm{T}_{3}$ - Aplicação de inseticida/ nematicida a base de benfuracarbe, na dose de $2 \mathrm{Kg}$ i.a. ha ${ }^{-1}$; $\mathrm{T}_{4}$ - Aplicação de herbicida a base de tebuthiuron, na dose de $1 \mathrm{Kg}$ i.a. ha ${ }^{-1} ; \mathrm{T}_{5}$ - Aplicação de inseticida/nematicida a base de carbofuran, na dose de $2,345 \mathrm{Kg}$ i.a. ha ${ }^{-1}$ com a aplicação do herbicida a base de tebuthiuron, na dose de $1 \mathrm{Kg}$ i.a. ha ${ }^{-1}$; $\mathrm{T}_{6}$ - Aplicação de inseticida/nematicida a base de benfuracarbe, na dose de $2 \mathrm{Kg}$ i.a. ha- ${ }^{-1}$ com a aplicação do herbicida a base de tebuthiuron, na dose de $1 \mathrm{Kg}$ i.a. ha-1.

A aplicação de inseticidas/nematicidas e/ou herbicidas foram realizados da seguinte forma: em cada unidade experimental foram abertos sulcos simulando um plantio convencional e os inseticidas/nematicidas foram aplicados diretamente sobre os toletes, antes da cobertura dos sulcos. Posteriormente, os toletes foram cobertos com solo e o herbicida foi aplicado em cobertura com micropipeta, nas unidades experimentais nas quais foi designado mediante sorteio. Foram realizadas adubações de cobertura utilizando-se $1 \mathrm{~g}$ de adubo formulado 20-05-20 por aplicação, sendo a primeira aos 48 dias após o plantio (DAP) e a segunda 68 DAP.A irrigação do experimento foi realizada, diariamente, por aspersão, até a conclusão do mesmo.
O experimento foi submetido a duas avaliações, umas aos 45 e outra aos 90 DAP. Na primeira foram avaliadas altura, número de folhas e número de plantas daninhas por vaso. Na segunda, avaliou-se altura da parte aérea, número de folhas, número de plantas daninhas, massa fresca e seca da parte aérea; massa fresca do sistema radicular; população final total de nematoides vaso ${ }^{-1}$, obtida com o somatório dos espécimes nas raízes e no solo. Os levantamentos populacionais de nematoides compreenderam sub-amostras contendo solos e raízes, que foram conduzidas ao laboratório e processadas pelos métodos de Jenkins (1964) e Coolen e D’herde (1972), respectivamente. A contagem dos nematoides foi realizada em microscópio óptico, com câmara de Peters, determinando-se o número de espécimes por vaso.

O delineamento experimental utilizado foi o inteiramente casualizado, com seis tratamentos e quatro repetições. Os dados foram submetidos à análise de variância $(\mathrm{p}<0,05)$, no programa estatístico SISVAR e, quando significativo, as diferenças entre as médias foram comparadas pelo teste de Scott-Knott, ao nível de 5\% de significância (FERREIRA, 2008).

\section{Resultados e Discussão}

A altura das plantas (Tabela 1) aos 45 DAP dos tratamentos testemunha $(42,75 \mathrm{~cm})$, Tebuthiuron $(32,62 \mathrm{~cm})$ e Carbofuran $(33,87 \mathrm{~cm})$ foram estatisticamente menores que os tratamentos Benfuracarbe $(44,75 \mathrm{~cm})$, Tebuthiuron+Carbofuran $(45,33 \mathrm{~cm})$ e Tebuthiuron+Benfuracarbe $(46,25 \mathrm{~cm})$.

Tabela 1: Avaliação da altura, número de folhas e plantas daninhas aos 45 e 90 dias após a aplicação dos tratamentos.

\begin{tabular}{|c|c|c|c|}
\hline Tratamento & Altura (cm) & $\mathrm{N}^{\circ}$ de folhas $^{1}$ & $\begin{array}{c}\mathrm{N}^{\circ} \text { de plantas } \\
\text { daninhas }\end{array}$ \\
\hline \multicolumn{4}{|c|}{45 dias } \\
\hline Testemunha & $42,75 \mathrm{a}$ & $5,25 \mathrm{~b}$ & $2,50 \mathrm{~b}$ \\
\hline Tebuthiuron & $32,62 \mathrm{a}$ & $4,00 \mathrm{a}$ & $0,00 \mathrm{a}$ \\
\hline Carbofuran & $33,87 \mathrm{a}$ & $5,00 \mathrm{~b}$ & $2,00 \mathrm{~b}$ \\
\hline Benfuracarbe & $44,75 \mathrm{~b}$ & $4,75 \mathrm{~b}$ & $2,00 \mathrm{~b}$ \\
\hline Tebuthiuron+Carbofuran & $45,33 \mathrm{~b}$ & $4,00 \mathrm{a}$ & $0,00 \mathrm{a}$ \\
\hline Tebuthiuron + Benfuracarbe & $46,25 \mathrm{~b}$ & $4,50 \mathrm{a}$ & $0,75 \mathrm{a}$ \\
\hline $\mathrm{CV}(\%)$ & 16,38 & 5,25 & 15,36 \\
\hline \multicolumn{4}{|c|}{90 dias } \\
\hline Testemunha & $69,50 \mathrm{a}$ & $6,00^{\text {ns }}$ & $1,00^{\text {ns }}$ \\
\hline Tebuthiuron & $64,75 \mathrm{a}$ & 6,75 & 0,50 \\
\hline Carbofuran & $59,75 \mathrm{a}$ & 6,00 & 1,25 \\
\hline Benfuracarbe & $75,75 b$ & 6,75 & 1,25 \\
\hline Tebuthiuron+Carbofuran & $77,66 \mathrm{~b}$ & 6,50 & 0,50 \\
\hline Tebuthiuron+Benfuracarbe & $77,25 \mathrm{~b}$ & 7,00 & 0,50 \\
\hline $\mathrm{CV}(\%)$ & 9,80 & 4,10 & 19,73 \\
\hline
\end{tabular}

Resultados semelhantes foram observados nas variáveis alturas aos 90 DAP, massa fresca da parte aérea (MFPA) e massa seca da parte aérea (MSPA) (Tabelas 1 e 2). 
Tabela 2: Avaliação da massa fresca (MFPA) e massa seca (MSPA) da parte aérea, massa fresca da raiz (MFRA) e população de nematoides aos 90 dias após a aplicação dos tratamentos.

\begin{tabular}{lcccc}
\hline Tratamento & MFPA (g) & MSPA (g) & MFRA (g) & Nematoide por vaso \\
\hline Testemunha & $9,54 \mathrm{a}$ & $2,76 \mathrm{a}$ & $11,95^{\text {ns }}$ & $499,00 \mathrm{~b}$ \\
Tebuthiuron & $9,26 \mathrm{a}$ & $2,58 \mathrm{a}$ & 13,08 & $487,25 \mathrm{~b}$ \\
Carbofuran & $8,34 \mathrm{a}$ & $2,10 \mathrm{a}$ & 9,17 & $792,75 \mathrm{~b}$ \\
Benfuracarbe & $14,20 \mathrm{~b}$ & $3,58 \mathrm{~b}$ & 12,91 & $46,75 \mathrm{a}$ \\
Tebuthiuron+Carbofuran & $13,78 \mathrm{~b}$ & $3,51 \mathrm{~b}$ & 12,46 & $99,75 \mathrm{a}$ \\
Tebuthiuron+Benfuracarbe & $14,56 \mathrm{~b}$ & $3,70 \mathrm{~b}$ & 13,83 & $708,75 \mathrm{~b}$ \\
CV $(\%)$ & 18,07 & 21,52 & 24,68 & 24,86 \\
\hline
\end{tabular}

De forma similar, Girotto et al. (2011) confirmaram a eficiência fotossintética do Tebuthiuron na cultura da cana-de-açúcar, variedade SP 80-3280. Após a aplicação em pré-emergência de dois herbicidas inibidores da fotossíntese, tebuthiuron e atrazine ${ }^{4}$, o primeiro proporcionou maior redução na taxa de transporte de elétrons, quando comparado aos tratamentos com atrazine e testemunha. Esta reação reduz a fixação de $\mathrm{CO}_{2}$, a produção de ATP e $\mathrm{NADPH}_{2}$, os quais são elementos essenciais ao crescimento das plantas (BREITENBACH et al., 2001). Quando não degradados ou metabolizados, os herbicidas podem ser ativos em um ou mais locais de ação dentro da planta. Esses locais de ação são enzimas, proteínas ou outros locais onde o herbicida se liga e dificulta alguma função normal da planta (CARVALHO, 2004). Nesses locais começam a exercer o seu mecanismo de ação, que pode ser definido como a principal reação química ou física que é afetada no interior da célula, que resulta na alteração do crescimento da planta (VICTÓRIA FILHO; CHRISTOFFOLETI, 2004). Resultados diferentes foram publicados por Barela (2005), que trabalhando com a interação do herbicida pendimethalin ${ }^{5}$ e os nematicidas aldicarb ${ }^{6}$ e terbufós ${ }^{7}$, aplicados no plantio da cana-de-açúcar, constatou que a produção de biomassa fresca da cana-de-açúcar foi afetada aos 90 dias após a brotação (DAB). É importante destacar que o herbicida pendimethalin tem como mecanismo de ação a inibição da divisão celular, diferentemente do tebuthiuron, cujo mecanismo é inibição da fotossíntese, fatos que poderiam explicar os resultados contrastantes (OLIVEIRA JUNIOR, 2001; RODRIGUES; ALMEIDA, 2005; SILVA et al., 2007). Quanto ao número de folhas (Tabela 1), também é possível observar que aos 45 DAP os tratamentos com tebuthiuron, por sua vez, diferiram estatisticamente dos tratamentos que não receberam o herbicida. Nesse sentido, o volume de irrigação após a aplicação do herbicida pode ter interferido no desenvolvimento da planta, pois, segundo Chistoffoleti e López-Ovejero (2005), herbicidas de alta solubilidade, como tebuthiuron $\left(2.500 \mathrm{ppm}\right.$ a $\left.25^{\circ} \mathrm{C}\right)$, quando utilizados em períodos de alta precipitação pluviométrica, podem ser lixiviados, o que aumenta o potencial de fitotoxicidade para a cultura, por seletividade de posicionamento no solo, principalmen-

${ }^{4}$ ATRAZINA® 50 SC - Herbicida Seletivo, do Grupo Químico das Trazinas - Suspensão Concentrada, Atanor S.A. - TTE GRAL J.D Péron, 646, 1038 - Buenos Aires - Argentina.

${ }^{5}$ HERBADOX, Herbicida seletivo, de ação não sistêmica, do grupo químico das dinitroanilinas. BASF Corporation - Route 168 \&JJ Spur, Hannibal MO, 63401 - USA

${ }^{6}$ DIAFURAN 50, Inseticida e Nematicida sistêmico. Shiroishi 1-1 Kurosaki, Yahatanishi-ku, Kitakyushu-city - Fukuoka prefecture / Japan.

${ }^{7}$ COUNTER 150G, Inseticida-Nematicida Sistêmico, do grupo Químico Organofosforado. Servatis S.A. Rodovia Presidente Dutra, km 300,5 - Resende/RJ. te em solos arenosos. A quantidade de herbicida adsorvido depende de suas propriedades químicas, das características físico-químicas do solo, principalmente da superfície específica e da capacidade de troca catiônica (CTC) dos minerais da fração argila, da formulação, da dose aplicada do produto e das condições climáticas. Por isso, informações obtidas em determinados locais, quando extrapoladas para outras regiões de clima e de solos diferentes, apresentam sempre um valor relativo (BLANCO et al., 1983).

O número de plantas daninhas por vaso aos 45 DAP (Tabela 1) foi estatisticamente inferior em todos os tratamentos que receberam tebuthiuron. Porém, aos 90 DAP não houve diferença significativa. A dose utilizada de tebuthiuron no experimento foi de $1 \mathrm{~kg}$ de ingrediente ativo por hectare, conforme faixa recomendada por Andrei (2005), e tem, segundo Lorenzi et al. (1983), uma importância econômica de 20 a $40 \%$ de produtividade, devido à efetiva ação do produto no combate a mato-competição. As espécies de plantas daninhas encontradas no experimento foram Gamochaeta spicata (Lam.) Cabr. (Macela), Sida cordifolia L. (Guanxuma), Cyperus rotundus L. (Tiririca), Digitaria horizontalis Willd. (capim-colchão), Eleusine indica (L.) Gaertn. (Pé-de-galinha) e Euphorbia hirta L. (Erva-de-Santa-Luzia).

A população de Pratylenchus zeae (Tabela 2) dos tratamentos testemunha, Tebuthiuron, Carbofuran e Tebuthiuron+Benfuracarbe diferiram estatisticamente dos tratamentos Benfuracarbe e Tebuthiuron+Carbofuran, cujas médias destes foram, respectivamente, 46,75 e 99,75, enquanto daqueles, 499,00; 487,25; 792,75 e 708,75.A espécie P. zeae é uma das mais importantes na cultura da cana de açúcar, em várias regiões produtoras (MOURA et al., 1990; NOVARETTI et al., 1998a; DINARDO-MIRANDA et al., 2001b; SANTOS et al., 2012)e encontra-se disseminada por praticamente todas as regiões canavieiras. No noroeste do Paraná, por exemplo, os nematoides do gênero Pratylenchus foram constatados em $85 \%$ de 74 amostras de cana de açúcar avaliadas por Severino et al. (2010). Desse total, P. zeae foi encontrado em $73 \%$ do material avaliado.

Diante disso, é importante destacar que a população inicial de espécimes de juvenis de nematoides, antes da instalação do experimento, era de 120 por $100 \mathrm{~cm}^{3}$ de solo. Ao final do experimento (90 DAP), a população no tratamento testemunha era de 499 espécimes de $P$. zeae por vaso, demonstrando multiplicação dos mesmos nas raízes da cana, atestando sua suscetibilidade, confirmada por diversos autores (SEVERINO et al., 2010; SANTANA et al., 2012; SANTOS et al., 2012). Embora o comportamento dos nematoides nos tratamentos tenha sido inconstante, alguns trabalhos demonstram a eficiência desses produtos na redução de populações de nematoides em cana (DINARDO-MIRANDA et al., 1996; NOVARETTI et al., 1998b). Dinardo-Miranda et al. (2001) testaram a ação dos nematicidas carbofuran, aldicarb e ethoprophos ${ }^{8}$ aplicados no plantio da cv. RB835113 em áreas infestadas por $M$. incognita e P. zeae. Todos os nematicidas mostraram-se eficientes no controle dos nematoides, reduzindo significativamente suas populações durante, pelo menos, quatro meses após o plantio. Com a redução populacional dos nematoides foram obtidos incrementos significa-

${ }^{8}$ RALZER 50 GR, Inseticida, nematicida sistêmico do grupo químico Metilcarbamato de Benzofuranila.Marunouchi 2 - chome 5-2, Chiyoda-ku, Tokyo 100-0005 Japão. 
tivos de produtividade agrícola, variando de 29,8 a $40 \mathrm{t} \mathrm{ha}^{-1}$, nas parcelas tratadas com carbofuran ou aldicarb (BOND et al., 2000; DINARDO-MIRANDA; GARCIA, 2002; DINARDO-MIRANDA et al., 2008).

$\mathrm{O}$ tratamento com o nematicida Benfuracarbe apresentou resultados superiores ao tratamento testemunha em relação à massa seca e fresca da parte aérea, e também os melhores resultados na redução da população de nematoides, juntamente com o tratamento tebuthiuron +carbofuran (Tabela 2). Além desses resultados promissores, o aumento da produtividade em até $50 \%$ pode ser assegurado pela empresa fabricante (AGROLINK, 2014).

É importante salientar que foram observadas injúrias no desenvolvimento inicial da cultura aos 15 DAP apenas para o tratamento Tebuthiuron+Carbofuran. Os sintomas acentuaram-se até os 45 DAP e regrediram a partir daí. Tais observações coadunam-se às de Barela e Christoffolet (2006), que também evidenciaram em seu trabalho com diferentes herbicidas e nematicidas, que no inicio do desenvolvimento da cultura 15 DAP todos os herbicidas estudados foram menos seletivos à cultura, quando na presença de algum nematicida, porém os graus da interação foram variáveis. Os resultados continuaram sendo avaliados aos 30,60 e 90 DAP e puderam detectar que os sintomas de fitotoxicidade diminuíram em relação às avaliações iniciais, demonstrando uma recuperação satisfatória das plantas, igualando-se estatisticamente para a variável produtividade.

$\mathrm{O}$ primeiro relato envolvendo fitotoxicidade de herbicidas associados a nematicidas, no Brasil, foi feito por Blanco et al. (1980), que descreveram a ocorrência de graves sintomas em canaviais da Usina São José, em Macatuba, SP, tratados com carbofuran no sulco de plantio, e tebuthiuron em pré-emergência das plantas daninhas, logo após o plantio. Para completar os estudos, estes autores conduziram testes em vasos e confirmaram que plantas tratadas com carbofuran e tebuthiuron, no mesmo ciclo, podiam apresentar severos sintomas de fitotoxicidade, caracterizados pela paralisação do crescimento, clorose do limbo foliar, requeima das folhas, começando pelo ápice e laterais das folhas e estendendo-se para a nervura central, ocorrendo, em alguns casos, secamento total da planta. Posteriormente, Copersucar (1982b) também relatou a presença de plantas com sintomas acentuados de fitotoxicidade, em canaviais comerciais tratados com o nematicida carbofuran e o herbicida tebuthiuron. Em contrapartida, Romão (2008b) avaliou o efeito do herbicida tebuthiuron com o nematicida carbofuranem cana-de-açúcar e não identificou interação entre ambos, porém, relatou um efeito fitotóxico do tebuthiuron, detectado apenas com equipamentos de alta sensibilidade.

\section{Conclusão}

O número de folhas foi afetado pela aplicação de Tebuthiuron aos 45 DAP, combinado ou não, demonstrando fitotoxicidade na cultura. Quanto à massa fresca e seca da parte aérea, avaliada apenas aos 90 DAP, os tratamentos com melhores resultados foram o Benfuracarbe, Tebuthiuron+Benfuracarbe e Tebuthiuron+Carbofuran, demonstrando a ausência de interação antagônica entre as combinações de Tebuthiuroncom os nematicidas utilizados. Os tratamentos Benfuracarbe e Tebuthiuron+Carbofuran foram eficientes no controle de $P$. zeae. Por fim, éimportante ainda salientar que foram observadas, visualmente, injúrias severas nos 15 primeiros DAP, nos tratamentos de Tebuthiuron+Carbofuran, levando a concluir que no inicio do desenvolvimento da cultura, os efeitos de uma possível interação podem ter sido potencializados, provavelmente pela fragilidade da cultura nessa fase.

\section{Referências}

AGROLINK. Pottente. IHARA, Ribeirão Preto, 01 ago. 2014. Disponível em: http://www.agrolink.com.br/ noticias/pottente--novo-produto-da-ihara-e-destaque-noinsectshow-2014_201053.html.Acesso em: 08 nov. 2014

ANDREI, E. Compêndio de defensivosagrícolas. Guia prático de produtos fitossanitários para uso agrícola. 7. ed. São Paulo: Andrei, 2005.

\section{BARELA, J.F. Seletividade de herbicidas para a} cultura da cana-de-açúcar (Saccharum spp.) afetada pela interação com nematicidas aplicados no plantio. Piracicaba, 2005. 82f. Dissertação (Mestrado) - Escola Superior de Agricultura “Luiz Queiroz".

BARELA, J. F.; CHRISTOFFOLETI, P. J. Seletividade de herbicidas aplicados em pré-emergência da cultura da canade-açucar (RB 867515) tratada com nematicidas. Planta Daninha,Viçosa, v. 24, n. 2, p.371-378, 2006.

BLANCO, H. G. et al. Fitotoxicidade em cana-de-açúcar causada pela interação de inseticida e herbicida residual. $\mathbf{O}$ Biólogo, São Paulo, v. 46, n. 10, p. 235-240, 1980a.

BLANCO, H. G. et al. Fitotoxicidade em cana-de-açúcar causada pela interação de inseticida e herbicida residual. O Biólogo, São Paulo, v.46, n. 10, p. 235-240, 1980 b.

BLANCO, H. G. et al. Persistência do herbicida metribuzin em solos cultivados com soja. Pesquisa Agropecuária Brasileira, Brasília, v. 18, p. 1073-1084, 1983.

BOND, J. P.; MCGAWLEY, E.C.; HOYS, J.W. Distribution of plant-parasitic nematodes on sugarcane in Louisiana and efficacy of nematicides. Journal of Nematology, Hanover, v.32, 4S, p.493-501, 2000.

BREITENBACH, J.; ZHU, C.; SANDMAN, G. Bleaching herbicide norflurazon inhibits phytoenedesaturase by competition with the cofactors. Journal of Agricultural and Food Chemmistry, Washington, v. 49, p. 5270-5272, 2001 .

CARVALHO, J. C. Mecanismo de ação dos herbicidas e sua relação com a resistência a herbicidas. In: CHRISTOFFOLETI, P. J.; OVEJERO, R. F. L.; CARVALHO, J. C. Aspectos de resistência de plantas daninhas a herbicidas. 2. ed. Campinas: Associação Brasileira de Ação a Resistência de Plantas aos Herbicidas, 2004. p.22-44. 
CHRISTOFFOLETI, P. J.; LÓPEZ-OVEREJO, R. F. Dinâmica dos herbicidas aplicados ao solo na cultura da cana-de-açúcar. Piracicaba: BASF, 2005. 49p.

CONAB. Companhia Nacional de Abastecimento. Acompanhamento da safra brasileira de cana-de-açúcar, terceiro levantamento, Brasília, p. 1-15, dez. 2013.

COOLEN, W. A.; D'HERDE, C. J. A method for the quantitative extraction of nematoides from plant tissue. Ghent: State Nematology and Entomology Research Station, Belgium, 1972. 77 p.

COPERSUCAR. Nematoides parasitos da cana-de-açúcar e seu controle. In: SEMINÁRIO DE TECNOLOGIA AGRONÔMICA, I, Piracicaba, SP, Anais... 1982a. p. 133153.

COPERSUCAR. Nematoides parasitos da cana-de-açúcar e seu controle. In: SEMINÁRIO DE TECNOLOGIA AGRONÔMICA, I, Piracicaba, SP, Anais... 1982b. p.133153.

DINARDO-MIRANDA, L. L.; FERRAZ, L. C. C. B. Patogenicidade de Pratylenchus brachyurus e P. zeaea duas variedades de cana-de-açúcar. Nematologia Brasileira, Piracicaba, v.15, p.9-16, 1991.

. Comportamento de variedades de cana-de-açúcar em relação a Meloidogyne javanica, em condições de campo. Nematologia Brasileira, Piracicaba, v. 19, p. 60-66, 1995.

Comportamento de genótipos de cana-de-açúcar em relação a Pratylenchus zeae. Nematologia Brasileira, Piracicaba, v. 20, n. 2, p. 52-58, 1996a.

Comportamento de genótipos de cana-de-açúcar a Pratylenchus zeae. STAB, Açúcar, Álcool e Subprodutos, Piracicaba, v. 17, n. 2, p. 39-41, 1996 b.

Reação de variedades de cana-de-açúcar a Pratylenchus zeae. STAB, Açúcar, Álcool e Subprodutos, Piracicaba, v. 17, n. 2, p. 39-41, 1998.

Efeitos da interação entre nematicidas e herbicidas em cana-de-açúcar.Nematologia Brasileira, Piracicaba, v. 25, n. 2, p. 197-203, 2001a.

. Efeitos da interação entre nematicida e herbicida em cana-de-açúcar. Nematologia Brasileira, Piracicaba, v. 25, n. 2, p. 197-203, 2001b.

.Efeitos da interação entre nematicida e herbicida em cana-de-açúcar. Nematologia Brasileira, Piracicaba, v.25, n. 2, p.197-203, 2001c.

DINARDO-MIRANDA, L. L.; GARCIA, V. Efeito da época de aplicação de nematicidas em soqueira de canade-açúcar. Nematologia Mediterranea, Bali, v. 26, n. 2, p. 65-67, 2002.
DINARDO-MIRANDA, L. L.; PIVETTA, J. P.;

FRACASSO, J. V. Influência da época de aplicação de nematicidas em soqueiras sobre as populações de nematóides e a produtividade da cana-de-açúcar. Bragantia, Campinas, v. 67, n. 1, p. 179-190, 2008.

FERREIRA, D. F. SISVAR: um programa para análises e ensino de estatística. Revista Symposium, Campinas, v. 6, p. 36-41, 2008

GARCIA, V.; SILVA, F. S.; DINARDO-MIRANDA, L. L. Comportamento de variedades de cana-de-açúcar em relação a Meloidogyne incognita. Revista Nacional do Álcool e Açúcar, Piracicaba, v. 17, n. 87, p. 14-19, 1997.

GIROTTO, M. et al. Eficiência fotossintética da cana-deaçúcar submetida à aplicação de atrazine e tebuthiuron em pré-emergência. Revista Brasileira de Herbicidas, Londrina, v. 10, p. 134-142, mai./ago. 2011.

JENKINS, W. R. A rapid centrifugal-flotation technique for separating nematodes from soil. Plant Disease Reporter, St Paul, 1964. 692p.

LORENZI, H. J. Plantas daninhas e seu controle na cultura da cana-de-açúcar.In: COPERSUCAR - REUNIÃO TÉCNICA AGRONÔMICA, Anais... 1983. p.59-53.

MOURA, R. M.; RÉGIS, E. M. O.; MOURA, A. M. Espécies e raças de Meloidogyne assinaladas em canade-açúcar no Estado do Rio Grande do Norte, Brasil. Nematologia Brasileira, Piracicaba, v.14, p. 33-38, 1990.

NOVARETTI, W. R. T. Efeitos de diferentes níveis de populações iniciais de Meloidogyne javanica em duas variedades de cana-de-açúcar (Saccharum spp.) cultivadas no Estado de São Paulo. Piracicaba, 1981. 100f. Dissertação (Mestrado em Agronomia) - Escola Superior de Agricultura "Luiz de Queiroz".

NOVARETTI, W. R. T.; MONTEIRO, A. R.; FERRAZ, L. C. C. B. Controle químico de Meloidogyne incognita e Pratylenchus zeae em cana-de-açúcar com carbofuran $\mathrm{e}$ terbufós. Nematologia Brasileira, Piracicaba, v. 22, n. 1, p. 60-73, 1998a.

NOVARETTI, W. R. T.; MONTEIRO, A. R.; FERRAZ, L. C. C. B. Controle químico de Meloidogyne incognita e Pratylenchus zeae em cana-de-açúcar com carbofuran e terbufós. Nematologia Brasileira, Piracicaba, v. 22, n. 1, p. $60-73,1998 b$

OLIVEIRA JUNIOR, R. S. Mecanismo de ação de herbicidas. In: OLIVEIRA JUNIOR, R.S; CONSTANTIN, J. (Coord.) Plantas daninhas e seu manejo. Guaíba: Agropecuária, 2001, p. 209-260.

PITELLI, R. A.; DURIGAN, J. C. Interferência de plantas daninhas em culturas agrícolas. Inf. Agropec., Belo Horizonte, v. 11, n. 129, p. 16-27, 1985. 
PROCÓPIO, S. O. et al. Manejo de plantas daninhas na cultura da cana-de-açúcar. Viçosa: Editora UFV, 2003. $150 \mathrm{p}$.

ROMÃO, R. V. Tebuthiuron com carbofuran na cultura da cana-de-açúcar. Presidente Prudente,2008a. $21 \mathrm{f}$.

Dissertação (Mestrado em Educação) - Universidade do Oeste Paulista.

ROMÃO, R. V. Tebuthiuron com carbofuran na cultura da cana-de-açúcar. Presidente Prudente, 2008b. 21f. Dissertação (Mestrado em Educação) - Universidade do Oeste Paulista.

RODRIGUES, B. N.; ALMEIDA, F. S. Guia de

herbicidas. 5. ed. Londrina: Editora dos autores, 2005.

$592 p$.

SANTANA, S. M. et al. Manejo de Pratylenchus zeae por plantas antagonistas, em solos de áreas de cultivo de canade-açúcar. Nematropica, Florida, v.42, p.63-71, 2012.

SANTOS, D. A. et al. Reaction of sugar cane genotypes to Pratylenchus brachyurus and P. zeae. Journal of Food, Agriculture \& Environment, Helsink, v.10. n. 2, p. 585587,2012

SANTOS, H. G. et al. Sistema Brasileiro de Classificação de solos. 3.ed. Brasília, DF: Embrapa, 2013. 353p.

SEVERINO, J. J.; DIAS-ARIEIRA, C. R.; TESSMANN, D. J. Nematodes associated with sugarcane in Sandy soils in Paraná, Brazil. Nematropica, Flórida, v.40, p.111-119, 2010a.

SEVERINO, J. J.; DIAS-ARIEIRA, C. R.; TESSMANN, D. J. Nematodes associated with sugarcane in Sandy soils in Paraná, Brazil. Nematropica, Flórida, v.40, p.111-119, 2010 b.

SILVA, A. A.; SILVA, J. F. Tópicos em manejo de plantas daninhas. Viçosa, MG: UFV. 2007. 367p.

VICTÓRIA FILHO, R.; CHRISTOFFOLETI, P. J. Manejo de plantas daninhas e produtividade da cana. Visão Agrícola, Piracicaba, v. 1, p. 32-37, 2004.

Recebido em: 04.05.2017 Aceito em: 05.09.2017 